\title{
Annotated BPMN Models for Optimised Healthcare Resource Planning ${ }^{\star}$
}

\author{
J. Bowles ${ }^{1}$, R. M. Czekster ${ }^{2}$, and T. Webber ${ }^{2}$ \\ 1 School of Computer Science, University of St Andrews \\ St Andrews KY16 9SX, UK \\ jkfb@st-andrews.ac.uk \\ 2 UNISC - University of Santa Cruz do Sul \\ 96815-900, Santa Cruz do Sul/RS, Brazil \\ \{ricardoc, thaiscs\} @unisc.br
}

\begin{abstract}
There is an unquestionable need to improve healthcare processes across all levels of care in order to optimise the use of resources whilst guaranteeing high quality care to patients. However, healthcare processes are generally very complex and have to be fully understood before enhancement suggestions can be made. Modelling with widely used notation such as BPMN (Business Process Modelling and Notation) can help gain a shared understanding of a process, but is not sufficient to understand the needs and demands of resources. We propose an approach to enrich BPMN models with structured annotations which enables us to attach further information to individual elements within the process model. We then use performance analysis (e.g., throughput and utilisation) to reason about resources across a model and propose optimisations. We show the usefulness of our approach for an A\&E department of a sizeable hospital in the south of Brazil and how different stakeholders may profit from a richer annotated BPMN-based model.
\end{abstract}

Keywords: Process Modelling, BPMN, Performance Analysis, Optimisation, Healthcare.

\section{Introduction}

Managers direct considerable efforts towards process modelling to understand complex behaviours in their application domains. Models, by themselves, are only useful if they enable the extraction of relevant and contextual information that yields process improvements (e.g. task order rearrangements, enhanced allocations, smart schedules, reduction of resources and so on). If not with optimisation in mind, models are mainly used for documentation, describing abstract representations of logical sequences of steps that must be executed in predetermined order to reach specific outcomes. The combination of behavioural modelling with performance evaluation $(\mathrm{PE})$ has recently received interest to handle

\footnotetext{
* This research was partially supported by EPSRC grant EP/M014290/1, RAE grant NRCP1617/5/62 and SFC grant SFC/AN/12/2017.
} 
complex processes in many application domains. PE uses techniques such as monitoring, analytical modelling or simulation to study systems and extract performance indicators (e.g. utilisation or queue length). These techniques help managers to fully understand processes and analyse performance metrics unveiling bottlenecks and more fitting options for resource allocations. One domain where findings can be particularly beneficial is the healthcare domain.

It is common practice today to use model notations such as Business Process Modelling \& Notation (BPMN)[18] to gain a better understanding of tasks and assigned resources (both machinery and personnel) required for different purposes across organisations. Although it is possible to apply performance evaluation techniques on such models, this is often not done due to lack of information on required resources for different tasks, inaccurate understanding of processes or simply a lack of knowledge required to understand what is needed as input for a comprehensive performance assessment. In the healthcare domain, the authors in [2] discuss the occurrence of failures when dealing with complex processes due to simple problems related to delivery of care by professionals. This could be avoided if problems were thoroughly analysed for performance problems using standardised notations. Resources (of any kind, e.g., supplies, machines, professionals and so on) are a key issue for maintaining high quality requirements to patients and so they must be addressed with utmost importance, not simply relying on process redesigns/reengineering approaches that permeate huge organisations with unconvincing results. Lack of flexibility in modelling is also a huge challenge for healthcare, since every hospital deals with different constraints and requirements. There must be some degree of adaptability when modelling and inferring performance [6]. However, simulation is sometimes viewed as an intricate technique due to the technical skills required when modelling, executing scenarios, interpreting outputs or making reliable suggestions to other managers or stakeholders. A further source of concern when integrating healthcare domain with performance assessment relates to a communication gap between clinical staff and process analysts [13].

This paper aims to bridge the gap between business process modelling and performance evaluation through task annotations for resource management. The idea is to use text annotations objects in BPMN to automatically fill simulation scenarios with interesting input data. BPMN with structured annotations could be potentially applicable for the automatic generation of simulation models (where analysts could benefit from quantitative evaluation of what-if scenarios, e.g. those maximising throughput or resources utilisation), or even analytical models (where analysts could infer state-based behaviours, performance bottlenecks or possible deadlocks). We propose an easy-to-use structured general-purpose notation format for annotating BPMN models with relevant information for resource planning. In this paper, we apply the notation on a case study describing a simplified healthcare scenario. The critical situation faced by healthcare in Brazil justifies our focus on that domain [10], and hence our aim to improve healthcare processes for hospital management. However, this work can be applied more broadly to different settings and any kinds of processes and 
simulations. The main goal is a detailed analysis and comparison of resources through simulation with the goal to be able to identify ways of improving processes and reduce resources. In the processes used in hospitals, this can be to reduce the number of staff required at different units at different times of the day, on different days of the week or even to accommodate for needs at different times of the year. High-level stakeholders or non-performance analysts can thus benefit from this annotation structure to help guide performance analysts towards strategic and profitable process configurations. Our case study is based on real processes followed by a hospital located in the south of Brazil (HSB). We have obtained the details of HSB's A\&E process through a series of staff interviews and data from the underlying ERP (Enterprise Resource Planning) system. Our approach, and the results of the analysis carried out on the scenarios that can be automatically generated from our annotated BPMN models. In this case, we used it to reflect on how to potentially achieve a saving in staff resources required to still guarantee tolerable waiting times.

This paper is structured as follows: Section 2 describes general details concerning BPMN modelling and expected performance indices. The work is described in the context of related work in Section 3. Section 4 proposes a structured annotation format for domain users to enhance their BPMN models for simulation. Section 5 shows how the annotations can be used in an example of a process followed by one department of hospital HSB. We discuss the scenarios generated automatically from the annotated BPMN model, and the results of their simulation as well as how they can be used for resource planning. Section 6 presents final considerations and ideas for future work.

\section{$2 \quad$ BPMN and Performance Modelling}

Enriching models with text annotations for performance analysis is not new and there is a myriad of proposed notations and extensions for coping with model descriptions using Layered Queueing Networks (LQN), Stochastic Petri Nets (SPN), Coloured Petri Nets (CPN), Performance Evaluation Process Algebra (PEPA), Well Formed Networks (WFN), Stochastic Automata Networks (SAN) and so on. Processes, on the other hand, may be modelled using BPMN, Workflows, or Unified Modelling Language/Activity Diagrams (UML/AD), among others. The focus of our present research lies on adding simple textual attachments to process elements with performance related data for later analysis (by simulation or other technique), thus offering broader analysis possibilities for stakeholders. The added annotations of BPMN processes may lead to a variety of further possible analyses, where a general view is depicted in Fig. 1.

There is a need to bridge process models with performance models, however, research is lacking on how to perform such integration. The main objective of this work is to discuss how such integration may occur so analysts could use the proposed notation in real world settings helping decisions on resource capacity or other performance metric of interest or Key Performance Indicator (KPI) according to the contextual domain of application $[15,14]$. 


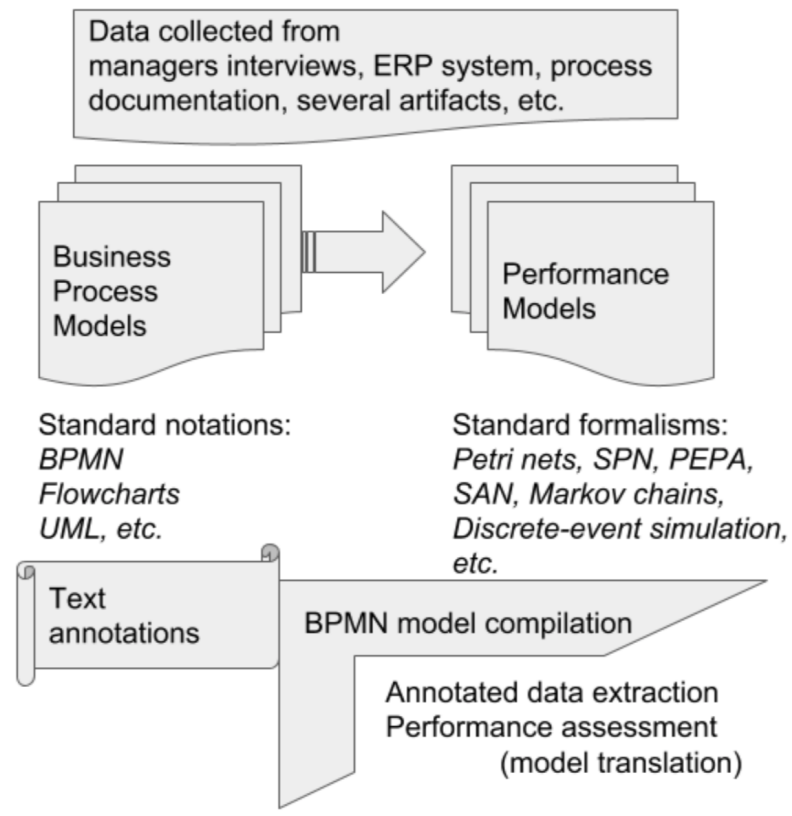

Fig. 1. Interplay between process models and performance models

Discrete Event Simulation (DES) is a well known performance evaluation technique with broad use amidst researchers, modellers, analysts, decision makers and managers in general $[22,19,12]$. Its scope encompasses different application domains and it is based on building a process model according to a system under study, assign probability distributions to arrivals, tasks durations and amount of needed resources and than execute the model throughout a replication length set up by the modeller. The idea is to compute the usual performance indexes e.g. throughput, utilisation, queue length, waiting time per task or resource and vary parameters for each scenario so comparisons may take place. Simulation is used when available data does not respect restrictions imposed by analytical modelling, for instance, exponential distributions, being more flexible to model patterns of behaviours through the process model [19]. We bring the benefits of this combination into a healthcare domain.

\section{The Context and Related work}

Text annotations in business models are not a novel approach to provide more unstructured detail for analysing processes. BPMN in itself uses modelling primitives to convey process behaviours, acting as an effort to document operations for different communities (e.g. managers, factory floor workers, company CEOs and other stakeholders). However, whilst BPMN's standardised notation allows 
for fast shared understanding among different roles, performance analysis at this level is hampered by the simplicity of the models that can be captured.

Significant efforts towards the ability to blend performance parameters into BPMN models were conducted throughout the years, with considerable advances. For instance [2] has discussed one possible use of richer BPMN models for healthcare. It is a lightweight approach called PyBPMN (Performabilityenabled BPMN) that extends BPMN for simulation. This work is the closest to the approach taken in this paper. The textual notation used in [2] to represent performability (performance and reliability) is, however, rather unconventional. By contrast, our approach uses a straightforward textual notation, easily understood by managers but rich enough with important parameters for later simulation analysis. Another distinction is that the authors in [2] aimed a model execution using an approach known as eBPMN execution (a domain specific language that retains BPMN's semantic properties) [7], whereas our work has the potential to devise multiple simulation scenarios instead of merely process simulation according to a BPMN model. Thus, our objective is to extend textual annotations to derive simulation scenarios to enable us to understand the effect of varying resources and expected delays and be able to do this dynamically. To the best of our knowledge, this perspective was not discussed in other work.

Other approaches discuss how BPMN could be used to enhance analysis improving automation and dealing with variability, a problem concerning clinical pathways in hospitals [21] and directives on how to combine modelling and simulation altogether [5]. The approach described in [21] discusses that Activity Diagrams (ADs) or BPMN models do not appropriately capture specific clinical requirements, being insufficient and inefficient when addressing performance. To address this, the paper offers an annotation-based approach to deal with those issues, but it presents an unstructured approach with textual data that may or may not be used for further analysis. Conversely, [5] combines BPMN with Business Process Simulation (BPS), a novel approach with considerable limitations. By contrast, our approach uses BPMN to annotate models that can be used in verified and validated simulation software tools such as Arena [19], AnyLogic, ProModel, Simul8, JMT [4], queueing123 GNU/Octave package [17] and so on.

The approach taken by [9] also differs considerable from ours, because it assumes the creation of an intermediary model that stands between the initial AD and the load performance model at the back-end. In their approach, users need to generate another model and fill it with load parameters for stress testing or other quality measures. The model uses a notation similar to extensions provided by UML.

Finally, work using simulation in a healthcare domain has been discussed thoroughly by several authors $[12,20,11,3,5]$. In particular, Mandahawi [16] has addressed the use of a continuous improvement technique (Six Sigma) and combined it with DES for carrying out waiting time analysis in an A\&E department with interesting discussions.

As a standard notation, BPMN provides means to create so called extensions with specific sets of descriptions in order to capture elements not anticipated 
in the original notation core. Our approach, however, does not rely on such extensions because we would like to work with a more structured approach for detailing specific resource related information. Our idea is to define a simple set of elements - which we consider simpler than using extensions - suitable for a broad range of multiple scenario DES analysis and usable by high-level managers and stakeholders (i.e. domain experts) alike.

\section{Structured text annotation proposal for process models}

The key BPMN component used here is the annotation, e.g., a textual description allowed by the standard which can be associated to different model elements. These texts are then processed by external tools and used to create a simulation model allowing the composition of multi-parameter, i.e., what-if scenarios. Managers could inspect each scenario outcome and adjust resource capacity or other KPI of interest [15] according to the evaluation needs. Auxiliary tools are used to process the BPMN model extracting useful data suited for DES, e.g., replication length and number of replications, time schedules, mean service time for each resource type, initial simulation conditions (e.g. work in progress), interarrival times, total/maximum amount of entities performing model tasks and so on.

It is worth mentioning that expert opinions are crucial when modelling processes (especially for later simulation prospects), since specific service time distributions should be employed for approximating real settings and yielding valid results. The choice of a probability distribution could profoundly impact analysis and completely change suggestions on resource management, schedules and allocations. For example, using an exponential distribution (one parameter, i.e., average observed value) for inter-arrival times is completely different from using a normal (where parameters are the average and the standard deviation) or a triangular distribution (e.g., a distribution having a minimum value, a mode and a maximum value) which are more suitable for situations characterised by extreme lack of data [19]. This is usually neglected by process modellers, despite being of vital importance for a sound and reliable analysis. The analyst should consult domain experts for the provision of useful statistics that dictates appropriate probability distributions for tasks and events. These measurements are present in ERP databases and logs, sometimes needing extra effort for validating, transforming and extracting relevant information within the vast amounts of textual data that could be available.

Table 1 lists structured text annotations for use in BPMN by managers or analysts, where they could adjust parameters and use diverse processing tools or scripts for generating simulations or analytical models. We stress the fact that our approach contains the least number of parameters for a comprehensive simulation study. In this paper, the main elements for attaching structured annotations are pool/swimlanes, start event, tasks (activities) and exclusive gateways (decisions) according to a standard tag-based BPMN extension proposal. Our set of annotations were inspired by the input parameters offered by the DES soft- 
Table 1. Structured annotations for BPMN elements with examples

\begin{tabular}{|c|c|}
\hline a) Per swimlane & 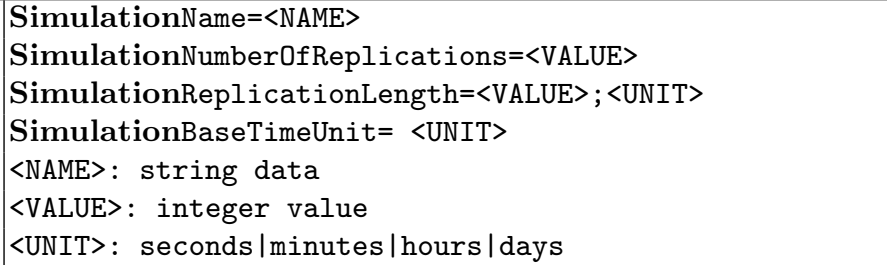 \\
\hline b) Per start event & 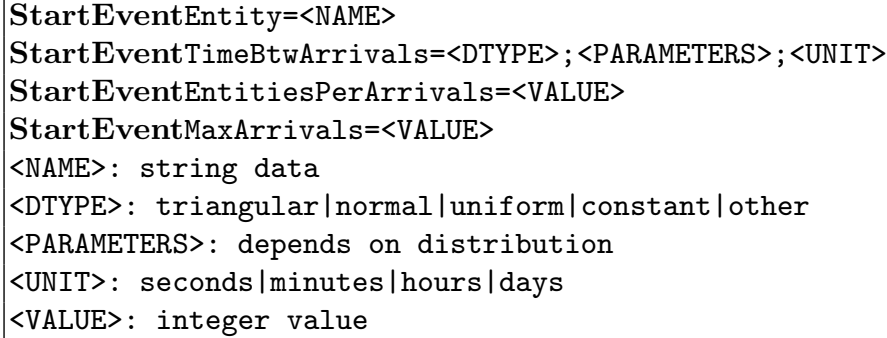 \\
\hline c) Per task event & 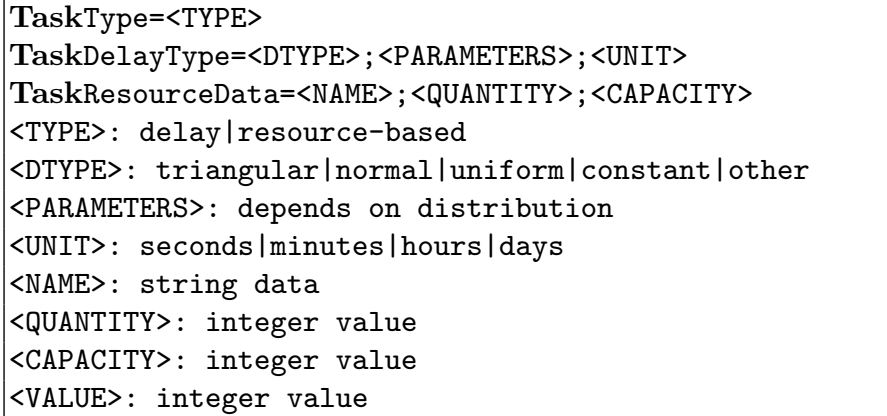 \\
\hline d) Per decision & $\begin{array}{l}\text { DecisionData }=\langle\text { TYPE }>\text {; }\langle\text { PERCENTAGES }>|<\text { CONDITIONS }> \\
<\text { TYPE }>\text { : probability lexpression (equation) } \\
\text { <PERCENTAGES }>\text { : set of comma separated values } \\
\text { <CONDITIONS }>\text { : logical expression (equation) }\end{array}$ \\
\hline
\end{tabular}

ware Arena [19]. The choice of this particular tool stems from the fact that it is widely used by a large community of practitioners, researchers and modellers.

At this point, we are devising the simulation scenarios from the annotated BPMN model and manually creating a process model in Arena. It is our aim, in future research, to incorporate the ability of creating models completely automatically. We have implemented a tool written in Java to support the scenario creation by opening BPMN models (with the annotations mechanism explained here) and parsing its standard XML file. This solution helps stakeholders understand which scenarios are possible as well as to visualise and select the best ones for execution (according to their requirements), using Arena to manually create the simulation model (though other DES could be used).

Table 1 defines the BPMN elements and the proposal of structured text annotations as follows: 
(a) The text annotations on pools (or specific swimlanes) specify global parameters related to simulation execution such as the NumberOfReplications for confidence intervals, the simulation time characterised by ReplicationLength and BaseTimeUnit. The later is an annotation to set the time unit for the calculated results.

(b) The start event element of BPMN models may append text annotations to specify the entities being analysed by their NAME (e.g., patients, clients, items). In addition, the simulation execution and the TimeBtwArrivals needs to be specified by its probability distribution type (i.e., triangular, exponential, normal, constant, and other for different expressions definitions, etc.) and its parameters (values for mean, standard deviation, mode and so on, according to type definitions) along with respective time units for the specified measures.

(c) Tasks are defined by their labels (i.e., NAME) and type (TYPE), whereby the type can only be delay or resource-based. If a task is resource-based, information about resources must be given in order to map the basic set of parameters for collecting performance indices related to queueing statistics and resource utilisation. A given resource has a label (i.e., an identification name), the quantity needed to perform the specific task and its capacity for the whole process execution (i.e., number of available resources with this label for the process).

(d) Exclusive gateways in the BPMN model representing decisions for taking specific flows can be more detailed using a structured annotation with its TYPE (i.e., indicated as probability values or an expression with logical conditions based on entity attributes, for example). For a probability type, a list of $1 . .(N-1)$ percentage values are needed for output flows of the gateway (where $N$ is the total number of output flows from a gateway).

Generic field names such as NAME are reserved for free text input, e.g., strings explaining some specific necessity or commentary describing some important task mention or desired behaviour. Fields containing a VALUE are composed of integers depending on the element they are located (in a swimlane, a start event or a task). The PARAMETERS found in StartEventTimeBtwArrivals and TaskDelayType labels indicate the values estimated for the time between arrivals and the average task duration, respectively, following the chosen probability distribution given in the field DTYPE.

The proposed format is suitable for models where those explained elements are present so modellers can use annotations to write proper simulation related tags for analysis. These annotations may be inserted in a manual fashion, however, an automatic tool could be effortlessly implemented to help users annotate the elements in a model avoiding mistakes or typos within the tags. Such a tool can save the annotations in the same model format (XML) to be opened and edited later, without interfering with the model's original set of elements and flow. We show how our approach has been used on a case study in the next section. 


\section{Case study: Brazilian hospital setting}

We apply our proposed annotated BPMN to a sizeable hospital HSB located at the southern state of Brazil. It is a hospital with approximately 200,000 occurrences per month (statistics from 2015), with 250 beds and 900 employees. It provides care to both public (under SUS - Brazilian universal public health system) and private patients. The hospital is located in a city with a population of around 160,000 with a further 100,000 living in the surroundings. The hospital is a regional reference for secondary care.

\subsection{An A\&E Process}

The A\&E department is viewed by management as the current bottleneck, as the resources are not evenly distributed, causing several delays and loss of revenue, despite dissatisfaction with the service. We have modelled this department using BPMN, and used our annotation mechanism to assign parameters to a future simulation model, where scenarios are to be created to demonstrate to management where the most critical deficiencies are. In addition, it serves as a way to identify further actions that should be implemented to improve the operation, reduce queues and waiting time for patients, as well as reduce costs and resources (balancing the utilisation among different professionals) to augment satisfiability with the service and perhaps increase revenue altogether by reducing unnecessary costs.

Fig. 2 contains the initial model for the A\&E department. In accordance with our previously introduced annotations, the pool has some general simulation parameters (shown at the top left-hand corner), the initial event (here Patient arrivals) has specific annotations related to simulation start rule, and all other tasks have annotations with resource related data. Non-critical patients arrive at $\mathrm{A} \& \mathrm{E}$ and go through an admission registering procedure (note that critical patients bypass this task and go straight to medical care/consultation). After registration, patients are classified according to their condition (Risk assessment process), which is performed by a Manchester Triage System trained nurse (with a given duration pattern according to the annotation). We stress the fact that the A\&E should be used only in critical cases, however, according to data observations, only $10 \%$ are in fact immediate care patients. The hospital management team recognises and is aware of this problem, but are unable to address this since they are obliged to provide care to every patient that arrives at the hospital. After classification patients go to the Medical consultation procedure, where a Clinical decision is made by the medical doctor to send the patient to a Medication \& Therapeutics procedure (with some routing probability, e.g. 50\%) or to Requiring LabTests procedure for a deeper investigation. Each possible flow from the exclusive gateway has its specific ending, i.e. patient discharge or patient care.

Fig. 2 also shows some desired scenarios envisaged by the analyst, making it possible to understand the impact of having different numbers of resources (such as the number of available medical doctors at a given moment in time) 


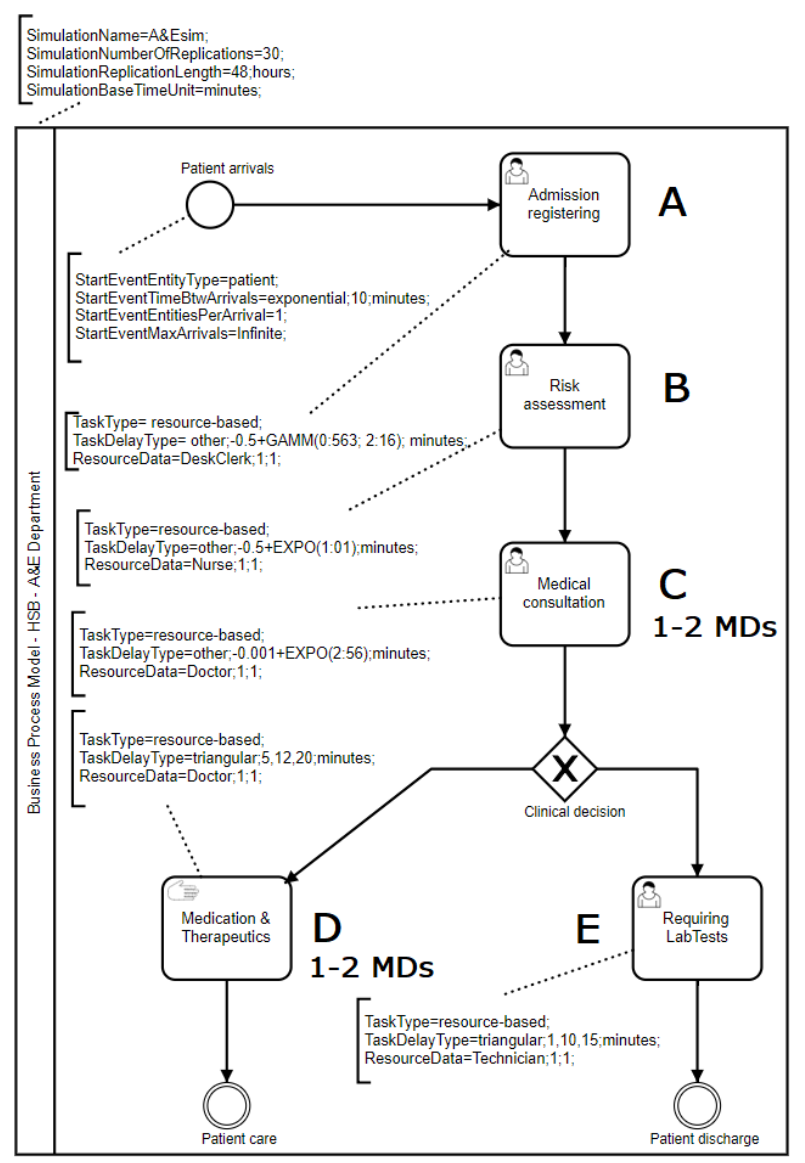

Fig. 2. Annotated BPMN model derived from HSB data for the A\&E process.

on performance. We have labelled the figure from A to E, and we have mapped some resource variations for our purposes, such as having one or two resources for tasks C and D (here Medical Doctors - MDs).

As the annotations show, we are mapping resource durations and scenarios for a simulation, as well as setting some important parameters that could be used by another (discrete event) simulation tool. It is important to notice that we are enriching a BPMN model, i.e., the resource-related data was not present in the original model given to us by hospital staff who are only able to describe the process flow and activities related to patients that come to the A\&E. Instead, we are proposing a format where data is available for a comprehensive simulation analysis where modellers are annotating tasks with probability distributions of interest as well as assigning numbers of resources, task durations and so on, so multiple scenarios can be automatically created with a reasonable amount of effort. For the creation of more simulation scenarios, we propose that modellers 
could use child annotations, e.g., annotations of annotations, as illustrated in Fig. 3.

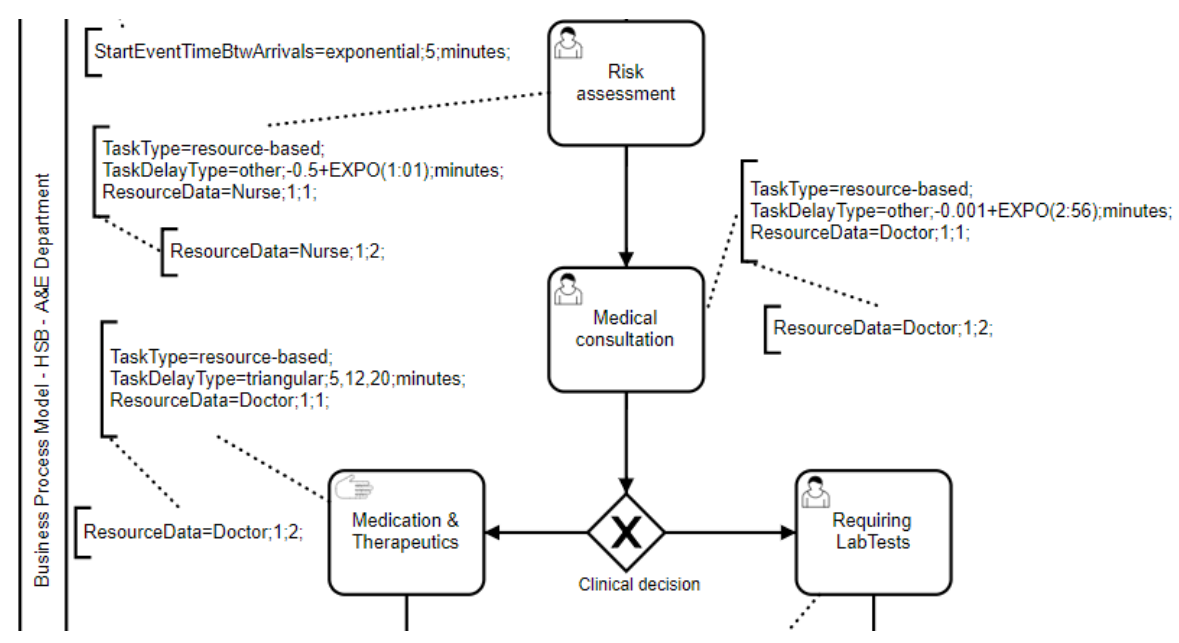

Fig. 3. Example of child annotations usage for deriving possible simulation scenarios.

Introducing yet another notation that modellers should be aware of is problematic and we are aware of this. However, we believe that an auxiliary user friendly tool could aid modellers specify annotations properly for later processing, as well as making it easier to check the consequences of changes to some of these annotations. A further advantage is that such a tool can create the intended set of scenarios for simulation automatically, and the DES tool of choice can then execute several simulations automatically where performance indices are calculated for posterior analysis. Going back to the original BPMN model, making changes to some of the annotations and parameters and rerunning simulations would give users a better understanding of their processes and the effects of changing resources at different points. Our approach combines strengths of BPMN and simulation, as it uses a straightforward mechanism to build simulation scenarios using annotations, a simple mechanism already present in the standard notation.

\subsection{Analysis Results}

We have used the annotation artifact as source to devise multiple scenarios for analysis. We have extracted the full annotations from the BPMN model by implementing a software written in the programming language Java which used XML APIs for parsing and working with models. The software identified BPMN elements such as pools/swimlanes, start and end events, tasks, sequence flow between tasks and exclusive gateways, and stored the full set of annotations 
in internal data structures. Then, the annotations are parsed to compute the amount of scenarios that would be created, using the textual variations and other data present in the model. Ideally, the analyst would select just a few scenarios to be executed from the potential high number of possible combinations of options that could be yielded by the user choices. Also, automatically created simulation models could be defined, where specific DES software could run in batch mode, notifying when the process has finished. At the moment, we are taking the scenarios generated by our software, selecting the most interesting ones and then manually creating the simulation models.

According to Fig. 2, the following scenarios are possible to be derived from the basic structured annotations:

- General simulation parameters (annotations at the pool element)

- Name (SimulationName): A\&Esim

- NumberOfReplications: 30

- ReplicationLength: 48 hours

- BaseTimeUnit (for reporting): minutes

- Start event element (annotations at the StartEvent element)

- EntityType: Patient

- TimeBtwArrivals: it follows an exponential distribution with parameter equal to 10 minutes

- Further parameters are irrelevant for the present analysis

- Task elements include (annotations at Task elements)

- Admission registering (A), Risk assessment (B), Medical consultation (C), Medication \& Therapeutics (D), Requiring LabTests (E). Different parameters are embedded within each annotation, with some variations as to the number of needed resources per task

Looking at the possible scenario variations for this model according to the annotations, we can see that the ResourceData parameter is different for some tasks. This represents the amount of resources the manager envisioned to analyse, i.e., the impact of these variations in the performance indices.

In this case, we have four scenarios, where the overall model follows the pattern Patient Arrival -- Process -- Exit, where Patient Arrival has no variations and Exit is just a sink (e.g. where all patients end). Note that we are disregarding the exclusive gateway in this analysis because it has no annotations of type and exit percentages in this example. For simulation purposes we assume $50 \%$ chance in the exclusive flows.

The four selected scenarios are as follows (see Fig. 2 - MD stands for Medical Doctor):

1. A (1 DeskClerk) - B (1 Nurse) - C (1 MD) - D (1 MD) - E (1 Technician)

2. A (1 DeskClerk) - B (1 Nurse) - C (2 MDs) - D (1 MD) - E (1 Technician)

3. A (1 DeskClerk) - B (1 Nurse) - C (1 MD) - D (2 MDs) - E (1 Technician)

4. A (1 DeskClerk) - B (1 Nurse) - C (2 MDs) - D (2 MDs) - E (1 Technician) 
Due to observed arrival rates, for this particular analysis we consider Task A to have no concerns, seeming well adjusted according to patient's inflow. In this analysis, we are concerned with investigating the influence on the number of MDs on the performance indices, so our scenarios will vary the amount of doctors at stations $\mathrm{C}$ and $\mathrm{D}$. It is worth mentioning that if one resource becomes $i d l e$, it may be shared for better performance - the DES software tool usually implements this behaviour automatically, because in the model we are creating resources of the same type, i.e., generic MDs.

It is noticeable that, depending on the choices made by the analyst while defining the annotations, the number of scenarios could be very large. For example, if one selects three distinct inter-arrival times, with a task with one resource associated having two parameter variations and another task with three resource quantities $(1,2$ and 4$)$, the number of total scenarios for this case is $3 * 2 * 3=18$ scenarios, which is a significant amount for a comprehensive analysis. In these cases, the analyst could manually reduce the selected parameter variations or use the tool to generate the full set of scenarios and then select the ones he or she wishes to study more thoroughly (only those would be executed).

Table 2 presents the simulation results for the generated scenarios using Arena [1]. We have extracted the main performance indices for 30 replications, with 48 hours duration and interarrival time per patient consisting of $10 \mathrm{~min}$ utes. For this BPMN model, the simulation model conversion was straightforward since no particular Arena element was used. We have used service and arrival times obtained from actual HSB data, with distribution fittings (using Arena's internal tool named InputAnalyzer). The tool has yielded the following parameters:

- Time between arrivals: 1 patient, on average, arrives every 10 minutes (we are modelling the busiest hours, e.g., from 10.00 to 14.00 of a weekday);

- A: Service time $-0.5+\operatorname{GAMM}(0.563,2.16)$ minutes;

- B: Service time $-0.5+\operatorname{EXPO}(1.01)$ minutes;

- C: Service time $-0.001+\operatorname{EXPO}(2.56)$ minutes;

- D: Service time TRIA $(5,12,20)$ minutes;

- E: Service time TRIA $(1,10,15)$ minutes.

Waiting Time (W) encompasses the time spent in queue plus the time under service (in Arena, this is called Total Time). For this metric, we have computed the average value for all replications.

Utilisation (U) considers the fraction of time that resources remain in Idle state (instead of Busy, i.e. attending patients), and it is computed internally by Arena. Adding resources would invariably impact performance indices positively (particularly utilisation). For managers, however, it implies additional costs that sometimes are prohibitive, and instead other alternatives should be taken into account (e.g. improving service times or addressing bottlenecks on other stations).

It is worth noticing that Scenario 2 has interesting utilisation levels for the medical doctor resources, i.e., $43.9 \%$ for doctor-C-D and $62.7 \%$ for doctor-E. It was not clear that this scenario would yield this outcome before our analysis, 
Table 2. Results for the simulation scenarios set by the analyst in the annotations.

\begin{tabular}{|c|c|c|c|c|}
\hline Scenario & Resource & $\begin{array}{c}\text { Utilisation } \\
\mathrm{U}(\%)\end{array}$ & $\begin{array}{c}\text { Waiting Time } \\
\text { W (minutes) }\end{array}$ & $\begin{array}{l}\text { Population } \\
\mathrm{N} \text { (patients) }\end{array}$ \\
\hline 1 & \begin{tabular}{l|} 
DeskClerk-A \\
Nurse-B \\
Doctor-C \\
Doctor-D \\
Technician-E
\end{tabular} & $\begin{array}{c}7.5 \\
6 \\
86.2 \\
62.6 \\
12.4\end{array}$ & 48.4 & $\approx 2.5$ (Consultation) \\
\hline 2 & $\begin{array}{l}\text { DeskClerk-A } \\
\text { Nurse-B } \\
\text { Doctor-C } \\
\text { Doctor-D } \\
\text { Technician-E }\end{array}$ & $\begin{array}{c}7.5 \\
7.5 \\
43.9 \\
62.7 \\
13\end{array}$ & 23.8 & negligible \\
\hline 3 & \begin{tabular}{l|} 
DeskClerk-A \\
Nurse-B \\
Doctor-C \\
Doctor-D \\
Technician-E
\end{tabular} & $\begin{array}{c}7.5 \\
7.4 \\
85.6 \\
30.7 \\
12.2 \\
\end{array}$ & 45.8 & $\approx 4.1$ (Consultation) \\
\hline 4 & \begin{tabular}{l|} 
DeskClerk-A \\
Nurse-B \\
Doctor-C \\
Doctor-D \\
Technician-E
\end{tabular} & \begin{tabular}{c|}
7.5 \\
7.6 \\
43.9 \\
31.1 \\
13.2 \\
\end{tabular} & 19.3 & negligible \\
\hline
\end{tabular}

and it just required one additional resource given the workload required. Also, the waiting time is affected by the number of resources, where Scenario 3 has the worst, despite the increase in terms of medical doctors. For the population metric, for Scenarios 2 and 4, no queueing took place, however, for Scenarios 1 and 3 , for the Consultation task, queue lengths of $\approx 2.5$ and $\approx 4.1$ respectively were calculated by the software, which is interesting, because Scenario 3 for instance has had an increase in terms of resources and still has formed significant patient queueing.

Our approach described here allows managers to annotate models and assign parameters for resources (and other measures) in a simple way, yielding performance indices for analysis and scenario comparisons. It would be sufficiently easy to derive parameters as needed, for the same model (e.g. we are considering static models as of now). We chose not to use too many parameter variations due to the number of potential scenarios that could be created. In future work, we will explore how to devise a mechanism to help select a set of interesting scenarios. 


\section{Final considerations}

Performance evaluation directly from BPMN models is not readily available for analysts and stakeholders. At present, process models and performance models are two distinct approaches with separate sets of primitives. This paper tackles this problem by providing an alternative where process models are enriched with textual annotations simple enough to be used by stakeholders with different backgrounds, but still powerful enough to provide interesting information for simulation. The structured annotations that can be attached to process elements include performance data relevant for creating different parameter scenarios, simulation execution, or analytical modelling. If some parameter is missing, our compiler uses predefined values to guarantee an initial analysis. Our approach has been used to tackle resource requirements within complex models to facilitate the informed revision and optimisation of healthcare processes.

In future work, we aim to extend the notation to encompass other advanced modelling and execution as well as devising a scenario report for users where they are able to select scenarios of interest. We will also consider the integration with a simulation package to automatically execute scenarios and generate a graphical report with suggestions.

In another line of work, we are using BPMN to capture clinical guidelines for the treatment of chronic conditions [8]. For patients with multiple ongoing chronic conditions, aka multimorbidity, several guidelines have to be applied simultaneously. We have used constraint solvers to automatically detect inconsistencies between such guidelines and suggest alternatives in accordance to certain parameters. If we can integrate the present BPMN annotations in our BPMN models for clinical guidelines, we may be able to exploit the benefits of both approaches. We will explore this combination in future work.

\section{References}

1. Arena simulation. https://www . arenasimulation.com/, accessed: 2018-06-06

2. Antonacci, G., Calabrese, A., D'Ambrogio, A., Giglio, A., Intrigila, B., Ghiron, N.L.: A BPMN-based automated approach for the analysis of healthcare processes. In: Proceedings of the 25th International Conference on Enabling Technologies: Infrastructure for Collaborative Enterprises (WETICE). pp. 124-129. IEEE Computer Society (2016)

3. Baril, C., Gascon, V., Miller, J., Côté, N.: Use of a discrete-event simulation in a kaizen event: a case study in healthcare. European Journal of Operational Research 249, 327-339 (2016)

4. Bertoli, M., Casale, G., Serazzi, G.: JMT: performance engineering tools for system modeling. ACM SIGMETRICS Performance Evaluation Review 36, 10-15 (2009)

5. Bisogno, S., Calabrese, A., Gastaldi, M., Ghiron, N.L.: Combining modelling and simulation approaches: How to measure performance of business processes. Business Process Management Journal 22, 56-74 (2016)

6. Bocciarelli, P., D'Ambrogio, A., Giglio, A., Paglia, E., Gianni, D.A.: Transformation approach to enact the design-time simulation of BPMN models. In: IEEE 23rd International WETICE Conference. pp. 199-204. IEEE Computer Society (2014) 
7. Bocciarelli, P., D'Ambrogio, A., Paglia, E.: A language for enabling model-driven analysis of business processes. In: 2nd International Conference on Model-Driven Engineering and Software Development (MODELSWARD). pp. 325-332. IEEE Computer Society (2014)

8. Bowles, J., Caminati, M., Cha, S.: An integrated framework for verifying multiple care pathways. In: Eleventh International Symposium on Theoretical Aspects of Software Engineering (TASE). IEEE Computer Society (2017)

9. Costa, L.T., Czekster, R., de Oliveira, F.M., de M. Rodrigues, E., da Silveira, M.B., Zorzo, A.F.: Generating performance test scripts and scenarios based on abstract intermediate models. In: Proceedings of the 24th Int. Conf. on Software Engineering and Knowledge Engineering (SEKE'2012). pp. 112-117 (2012)

10. Doniec, K., Dall'Alba, R., King, L.: Brazil's health catastrophe in the making. The Lancet (July 2018)

11. Forsberg, H.H., Aronsson, H., Keller, C., Lindblad, S.: Managing health care decisions and improvement through simulation modeling. Quality Management in Health Care 20, 15-29 (2011)

12. Günal, M., Pidd, M.: Discrete event simulation for performance modelling in health care: a review of the literature. Journal of Simulation 4, 42-51 (2010)

13. Harper, P.R., Pitt, M.A.: On the challenges of healthcare modelling and a proposed project life cycle for successful implementation. Journal of the Operational Research Society 55, 657-661 (2004)

14. Ioan, B., Nestian, A.S., Tita, S.M.: Relevance of key performance indicators (kpis) in a hospital performance management model. Journal of Eastern Europe Research in Business \& Economics 2012, 1-15 (2012)

15. Khalifa, M., Khalid, P.: Developing strategic health care key performance indicators: A case study on a tertiary care hospital. Procedia Computer Science 63, 459-466 (2015)

16. Mandahawi, N.: Reducing waiting time at an emergency department using design for Six Sigma and discrete event simulation. International Journal of Six Sigma and Competitive Advantage 6(1/2), 91-104 (2010)

17. Marzolla, M.: The qnetworks toolbox: a software package for queueing networks analysis. In: International Conference on Analytical and Stochastic Modeling Techniques and Applications. LNCS, vol. 6148, pp. 102-116. Springer (2010)

18. OMG: Business Process Model \& Notation. v2.0. OMG, http://www.omg.org. (2011), http://www.omg.org, doc. id: formal/2011-01-03.

19. Rossetti, M.D.: Simulation Modeling and Arena. Wiley Press, 2 edn. (2010)

20. Shim, S.J., Kumar, A.: Simulation for emergency care process reengineering in hospitals. Business Process Management Journal 16, 795-805 (2010)

21. Shitkova, M., Taratukhin, V., Becker, J.: Towards a methodology and a tool for modeling clinical pathways. Procedia Computer Science 63, 205-212 (2015)

22. Sokolowski, J.A., Banks, C.M.: Principles of modeling and simulation: a multidisciplinary approach. John Wiley \& Sons (2011) 